Proc. 13th International School on Theoretical Physics: Symmetry and Structural Properties of Condensed Matter

\title{
Generation, Absorption and Photoconductivity in 2D Structures of Perovskite with Nanodisc Quantum Dots
}

 \\ ${ }^{a}$ Department of Physics and Medical Engineering, Rzeszów University of Technology, \\ al. Powstańców Warszawy 6, 35-959 Rzeszów, Poland
}

${ }^{b}$ Research and Development Centre for Photovoltaics, ML System S.A., Zaczernie 190G, 36-062 Zaczernie, Poland

\begin{abstract}
An array of semiconductor quantum dots introduced into photoelectric materials is currently an effective tool to increase the efficiency of photoelectric devices and photovoltaic cells. This is related to additional energy levels which expand the range of absorption frequencies towards the infrared. We consider the model with a semiconductor quantum dot in form of nanodisc surrounded by another semiconductor material. The results of calculation of the generation of non-equilibrium carriers in the conduction and valence bands are presented. The calculations account for discrete levels of the quantum dots embedded into perovskite material. Using the calculated generation and relaxation rates, the photocurrent is also determined.
\end{abstract}

DOI: 10.12693/APhysPolA.135.1287

PACS/topics: photovoltaic effects, quantum dots, perovskite solar cells

\section{Introduction}

Photovoltaic materials with perovskites [1] are of increasing use on an industrial scale now [2]. It is related to their extreme efficiency combined with rather cheap production process. In this process, the perovskite material can be easily applied by printing on various surfaces. The increasing efficiency of a cell can be reached by using some low-dimensional structures, which provide additional energy levels. It allows quantum transitions for a wide wavelength spectrum of electromagnetic radiation [3]. Numerous recent experiments confirm the existence of such effect [4].

For the calculation of light absorption and photocurrent, the energy levels and wave functions of localized states in the quantum dot (QD) should be determined. In most cases, for the description of electronic states in the quantum dot, one can use the single-band semiconductor model. An example is the II-VI quantum dot of CdTe (CdS), or the III-V dot (GaAs, AlGaAs, etc.). In the case of narrow-gap semiconductors, a multiband model should be used.

In the following consideration, a single-band model is used. We assume that the spin-orbit interaction is weak, and it can be omitted in the calculations.

\section{Model}

The model is described by the Hamiltonian on electron with effective mass $m_{c}$ in the conduction band of the semiconductor QD. The corresponding Schrödinger equation reads

*corresponding author; e-mail: wolan@prz.edu.pl

$$
\left[-\frac{\hbar^{2} \nabla^{2}}{2 m_{c}}+U(r)-E\right] \psi(\boldsymbol{r})=0 .
$$

The geometry of the system dot-environment is a twodimensional potential $U(r)$ with cylindrical symmetry (circular for 2D). The solution of Eq. (1) gives the energy levels in the quantum dot, which are presented in Fig. 1.

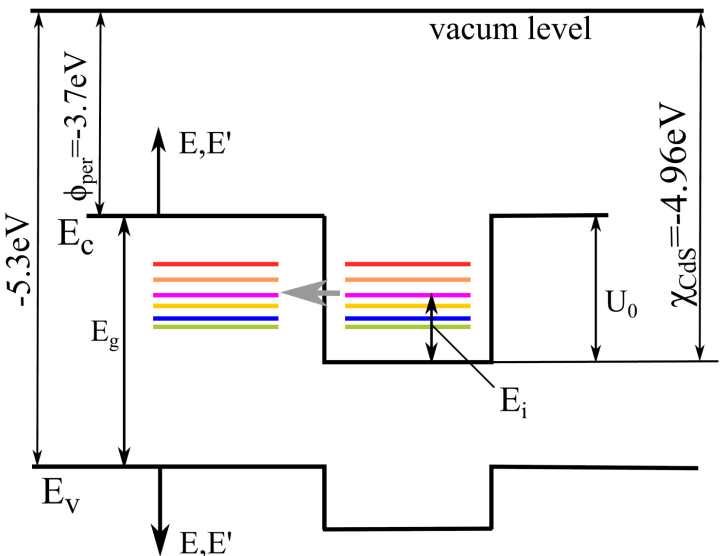

Fig. 1. Schematic presentation of the electronic structure of $\mathrm{QD}$ for CdS quantum dot in perovskite band structure and the band structure of adjacent quantum dots.

Generation rate of electrons in the conduction band due to electron excitations from the dot levels and from the valence band (here $E>0$ ) and the generation rate of holes (here $E<-E_{g}$ ) can be calculated by using

$$
\begin{aligned}
& g_{c}(E)=[1-f(E)]\left[N_{d} \sum_{i} f\left(E_{i}\right) \alpha_{i c}\left(E-E_{i}\right)\right. \\
& \left.+\int_{-\infty}^{-E_{g}} \mathrm{~d} E^{\prime} f\left(E^{\prime}\right) \tilde{\alpha}_{v c}\left(E, E^{\prime}\right)\right],
\end{aligned}
$$




$$
\begin{aligned}
& g_{v}(E)=f(E)\left[N_{d} \sum_{i}\left[1-f\left(E_{i}\right)\right] \alpha_{v i}\left(E_{i}-E\right)\right. \\
& \left.+\int_{0}^{\infty} \mathrm{d} E^{\prime}\left[1-f\left(E^{\prime}\right)\right] \tilde{\alpha}_{c v}\left(E, E^{\prime}\right)\right],
\end{aligned}
$$

where $N_{d}$ is the density of quantum dots, and $i=1,2, \ldots$ labels the energy levels in the dot.

The light absorption coefficients for transitions dot level-conduction (valence) band are

$$
\begin{aligned}
& \alpha_{i c}\left(E-E_{i}\right)= \\
& \quad \frac{2 \pi}{\hbar} \frac{4 \pi^{2} e^{2} \hbar^{2} P^{2} \mathcal{A}_{0}^{2}\left(E-E_{i}\right) \rho_{c}(E)}{m_{0}^{2} c^{2}} \\
& \quad \times \sum_{\boldsymbol{k}} \mid A \int_{0}^{R_{c}} r \mathrm{~d} r J_{m}\left(k_{n} r\right) J_{m}(k r) \\
& +\left.B \int_{R_{c}}^{\infty} r \mathrm{~d} r K_{m}\left(\kappa_{n} t\right) J_{m}(k r)\right|^{2} \delta\left(E-\varepsilon_{c \boldsymbol{k}}\right), \\
& \alpha_{v i}\left(E_{i}-E\right)= \\
& \quad \frac{2 \pi}{\hbar} \frac{4 \pi^{2} e^{2} \hbar^{2} P^{2} \mathcal{A}_{0}^{2}\left(E_{i}-E\right) \rho_{v}(E)}{m_{0}^{2} c^{2}} \\
& \quad \times \sum_{\boldsymbol{k}} \mid A \int_{0}^{R_{c}} r \mathrm{~d} r J_{m}\left(k_{n} r\right) J_{m}(k r) \\
& \quad+\left.B \int_{R_{c}}^{\infty} r \mathrm{~d} r K_{m}\left(\kappa_{n} t\right) J_{m}(k r)\right|^{2} \delta\left(E-\varepsilon_{v \boldsymbol{k}}\right),
\end{aligned}
$$

where $R_{c}$ is the radius of QD.

The interband absorption coefficients are calculated by using the golden rule

$$
\begin{aligned}
& \tilde{\alpha}_{v c}\left(E, E^{\prime}\right)= \\
& \frac{2 \pi}{\hbar} \sum_{\boldsymbol{k}}|\langle v \boldsymbol{k}|\hat{V}| c \boldsymbol{k}\rangle|^{2} \delta\left(E-E^{\prime}+\varepsilon_{v \boldsymbol{k}}-\varepsilon_{c \boldsymbol{k}}\right) \delta\left(E^{\prime}-\varepsilon_{v \boldsymbol{k}}\right) \\
& \tilde{\alpha}_{c v}\left(E, E^{\prime}\right)= \\
& \frac{2 \pi}{\hbar} \sum_{\boldsymbol{k}}|\langle v \mathbf{k}|\hat{V}| c \boldsymbol{k}\rangle|^{2} \delta\left(E^{\prime}-E+\varepsilon_{v \boldsymbol{k}}-\varepsilon_{c \boldsymbol{k}}\right) \delta\left(E^{\prime}-\varepsilon_{c \boldsymbol{k}}\right)
\end{aligned}
$$

For the calculation of energy relaxation time two mechanisms are of main importance: recombination to the valence (conduction) band and to discrete quantum dots levels. The other mechanisms are included by using phenomenological parameters

$$
\begin{gathered}
\tau_{c}^{-1}(E)=N_{d} \sum_{i}\left[1-f\left(E_{i}\right)\right] \alpha_{i c}\left(E-E_{i}\right) \\
+\int_{-\infty}^{-E_{g}} \mathrm{~d} E^{\prime}\left[1-f\left(E^{\prime}\right)\right] \tilde{\alpha}_{v c}\left(E, E^{\prime}\right)+\tau_{0 c}
\end{gathered}
$$

$$
\begin{gathered}
\tau_{v}^{-1}(E)=N_{d} \sum_{i} f\left(E_{i}\right) \alpha_{v i}\left(E_{i}-E\right) \\
+\int_{0}^{\infty} \mathrm{d} E^{\prime} f\left(E^{\prime}\right) \tilde{\alpha}_{c v}\left(E, E^{\prime}\right)+\tau_{0 v} .
\end{gathered}
$$

The density of generated photocarriers depends on the generation and relaxation rates. In stationary state we obtain

$$
\delta n(E)=g(E) \tau_{c}(E) .
$$

The photocurrent is due to the carriers reaching the $p-n$ junction, which is located at a distance $d$ from the region of electron excitation. If $d \gg \ell(\ell$ is the mean free path), then the current is due to diffusion of carriers. It can be calculated from

$$
j_{p h}=\frac{e}{d} \int_{0}^{\infty} \mathrm{d} E D_{c}(E) \delta n(E) .
$$

The set of these equations allow to calculate the main characteristics of photovoltaic devices.

\section{Results of calculations and conclusions}

Here we consider the generation, absorption and photocurrent in the structure with CdS quantum dot in $\mathrm{CH}_{3} \mathrm{NH}_{3} \mathrm{PbI}_{3-x} \mathrm{Cl}_{x}$ perovskite. Figures 2 and 3 present the dependence of absorption coefficients, relaxation rates, and generation of carriers as a function of energy in conduction and valence bands for two different Fermi level positions. The magnitude of calculated photocurrent and the parameters are included in the description of the figures.

As we see, the density of photocarriers generated by interband transitions is much larger than for the carriers generated in transitions between discrete dot levels and the bands. However, the real efficiency of quantum dots in the generation of charge carriers can be significantly greater. This is related to the broadening of energy levels due to the overlap of wave functions localized at the neighboring dots and due to the dispersion of dot sizes.

The calculated recombination factor in the quantum dot is of the order $\tau^{-1} \sim 10^{20} \mathrm{~s}^{-1}$. Taking it into account leads to very effective mechanism of recombination at the dot traps, which strongly reduces the photocurrent. However, one can show that the recombination at the dot can be blocked by the $p-n$ junction forming at the interface between the dot and perovskite solid. Because of that, it is possible to generate additional charge carriers from the energy levels lying in the perovskite energy gap.

\section{Acknowledgments}

This work is supported by the National Science Center in Poland as a research project No. DEC2017/27/B/ST3/02881 and partly by the National Center of Research and Development in Poland, project POIR.01.01.01-00-598/15. 

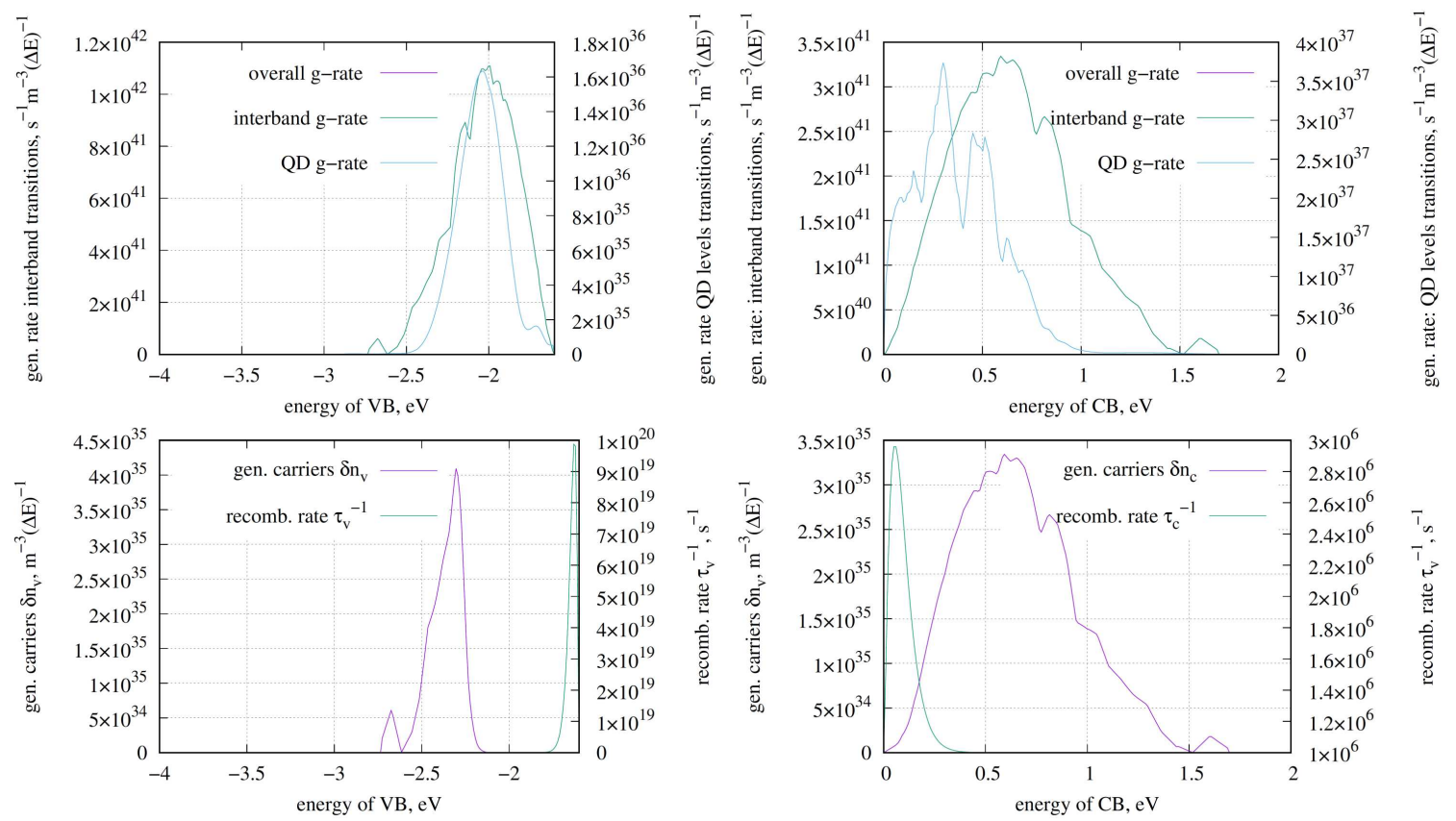

Fig. 2. Calculated generation and relaxation rates for CdS quantum dot on perovskite solid. The parameters of calculations are: chemical potential $\mu=-0.4 \mathrm{eV}$, temperature $T=300 \mathrm{~K}$, radius of $\mathrm{QD} R_{0}=4 \mathrm{~nm}$. Calculated currents for electrons and holes are: $j_{p h c}=6.761 \mathrm{~mA} / \mathrm{cm}^{2}$ and $j_{p h v}=5.355 \mathrm{~mA} / \mathrm{cm}^{2}$.
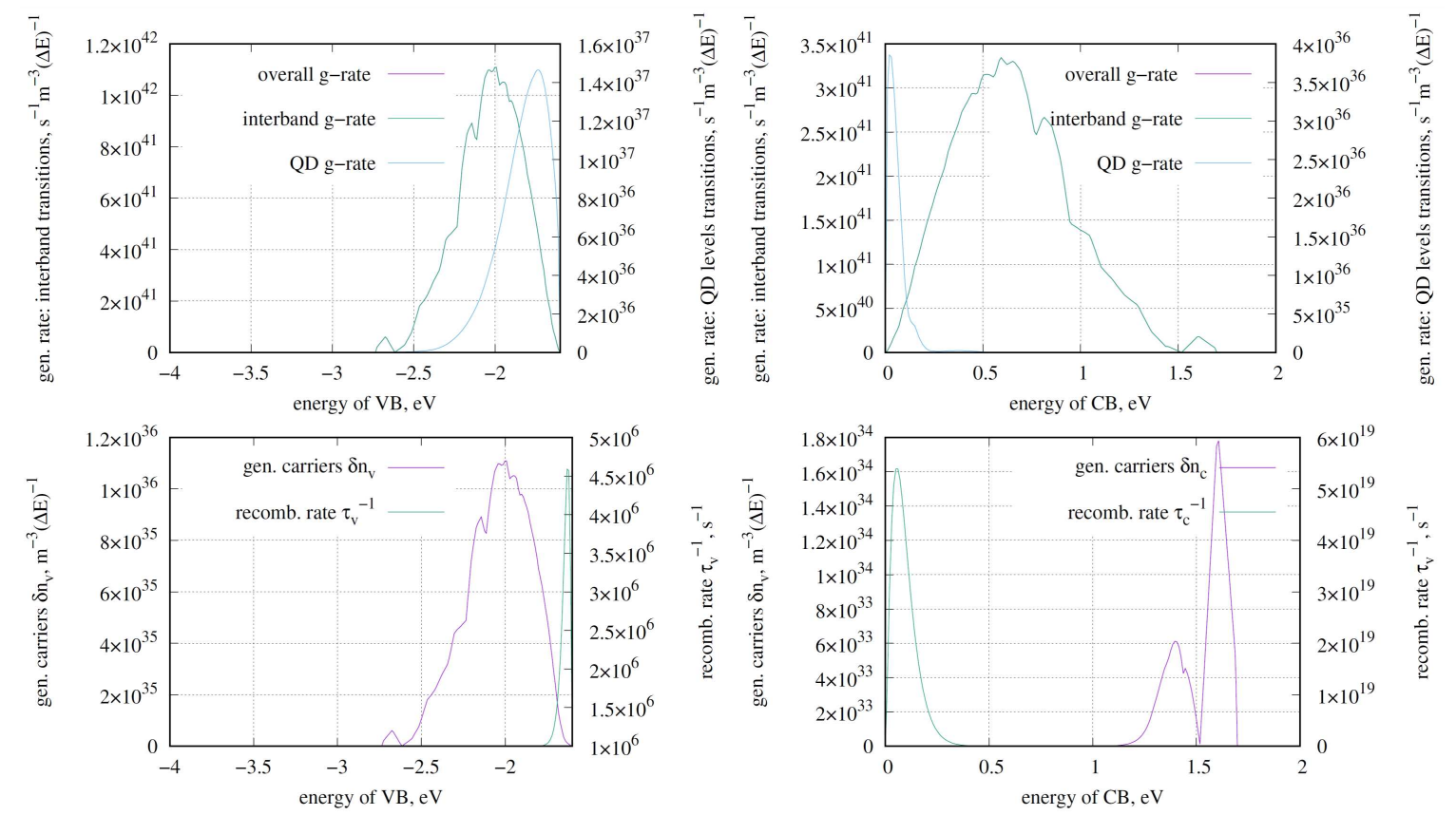

Fig. 3. Calculated generation and relaxation rates for CdS quantum dot on perovskite solid. The parameters of calculations are: chemical potential $\mu=-1.2 \mathrm{eV}$, temperature $T=300 \mathrm{~K}$, radius of QD $R_{0}=4 \mathrm{~nm}$. Calculated currents for electrons and holes are: $j_{p h c}=0.182 \mathrm{~mA} / \mathrm{cm}^{2}$ and $j_{p h v}=31.316 \mathrm{~mA} / \mathrm{cm}^{2}$.

\section{References}

[1] N.G. Park, Mater. Today 18, 65 (2015).

[2] T. Ibn-Mohammed, S. Koh, I. Reaney, A. Acquaye, G. Schileo, K. Mustapha, R. Greenough, Renew. Sustain. Energy Rev. 80, 1321 (2017).
[3] C. Liu, M. Hu, X. Zhou, J. Wu, L. Zhang, W. Kong, X. Li, X. Zhao, S. Dai, B. Xu, C. Cheng, NPG Asia Mater. 10, 552 (2018).

[4] S.M. Ali, S.M. Ramay, M.H. Aziz, N. ur Rehman, M. AlGarawi, S. AlGhamd, A. Mahmood, T.S. Alkhuraiji, S. Atiq, Org. Electron. 62, 21 (2018). 